\title{
Post-Natal Spontaneous Resolution of a Congenital Pulmonary Airway Malformation in an Infant: Plain Radiographic and CT Manifestations
}

\author{
John Amodio ${ }^{1 *}$, Agnes Perenyi ${ }^{2}$, Jonathan Zember ${ }^{2}$, Matthew Smith ${ }^{1}$ \\ ${ }^{1}$ Department of Radiology, SUNY Downstate Medical Center, New York, USA \\ ${ }^{2}$ Department of Pediatrics, SUNY Downstate Medical Center, New York, USA \\ Email: *John.Amodio@downstate.edu
}

Received January 20, 2012; revised April 26, 2012; accepted May 10, 2012

\begin{abstract}
We present a case of a near-term infant who developed respiratory distress, shortly after birth. Chest X-ray showed a right sided tension pneumothorax (PTX) with a large air containing structure at the base of the right hemithorax. The pneumothorax was partially evacuated with needle aspiration at which time the patient became asymptomatic, despite a small residual pneumothorax. CT scan of the chest confirmed a multilocular air-filled structure within the right lower lobe, most likely consistent with a congenital pulmonary airway malformation (CPAM). Subsequently, there was spontaneous resolution of the PTX and CPAM prior to surgical intervention. This case illustrates post-natal spontaneous resolution of a CPAM, thus obviating the need for surgical removal.
\end{abstract}

Keywords: Congenital Cystic Pulmonary Malformation; Spontaneous Resolution; Pneumothorax; Infant

\section{Introduction}

Congenital pulmonary airway malformation (CPAM), previously known as congenital cystic adenomatoid malformation (CCAM), is a rare developmental anomaly of the lower respiratory tract, in which a part of a lobe of lung is replaced by a non-working piece of abnormal lung tissue. Historically, some lesions have been described as having a "cystic" appearance, and composed of bronchial-like or bronchiolar-like structures in various "hamartomatous" arrangements, while others have had a more "adenomatoid" appearance pathologically. Patients with CPAM may present in the postnatal/infancy period with respiratory distress, or they may remain asymptomatic [1]. Spontaneous pneumothorax may be an uncommon clinical association with CPAM [2], but may be a cause of respiratory distress in patients with this anomaly.

While antenatal resolution of CPAM is well documented, post-natal spontaneous resolution of CPAM appears to be a rare event [3]. In this case, we present a near-term newborn who presented with spontaneous tension PTX associated with an underlying CPAM. After partial evacuation of the PTX, the patient became asymptomatic. Subsequently, spontaneous resolution of the CPAM occured, obviating the need for surgical interven-

"Corresponding author. tion.

\section{Case Report}

The patient was born by vaginal delivery, at 37 gestational weeks. The pregnancy was uncomplicated. No prenatal sonogram was performed.

Shortly after birth, the infant became tachypnic. Chest X-ray showed a right sided tension PTX with a large air containing structure at the base of the right hemithorax (Figure 1), thought to represent a congenital pulmonary airway malformation (CPAM).

Subsequently the right sided PTX was drained by needle aspiration. About $30 \mathrm{ml}$ air was removed. The infant became asymptomatic after partial evacuation of the pneumothorax. A repeat chest X-ray, after PTX aspiration, showed minimal residual PTX at the right base with a focal air filled structure at the right base (Figure 2), unchanged from prior images. The infant was closely monitored and remained asymptomatic.

As there was persistence of the right basilar abnormality, a chest CT scan with contrast was performed which showed multilocular air filled structures within the right lower lobe of the lung; the entire lesion measured approximately $3.4 \times 2.4 \mathrm{~cm}$ (transverse and AP dimensions respectively). The largest cyst measured $2 \mathrm{~cm}$. The lesion appeared to be at the periphery of the right lower lobe parenchyma, bordering the pleural surface (Figure 3). 


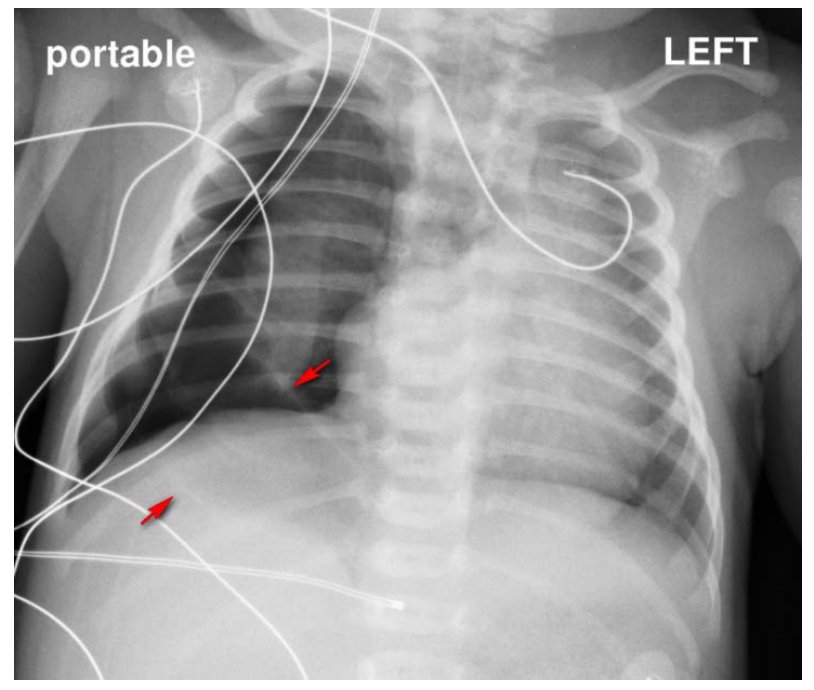

Figure 1. Frontal Chest Radiograph, demonstrates large right spontaneous pneumothorax with evidence of tension. There is leftward deviation of the mediastinum. A large air filled structure is noted at the right lung base (arrows).

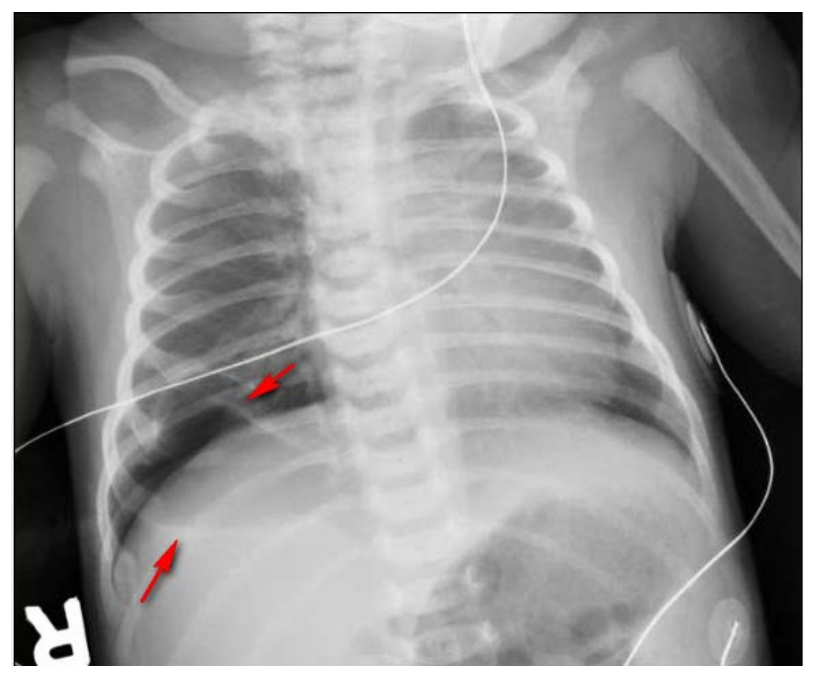

Figure 2. Frontal Chest Radiograph, demonstrates small residual right pneumothorax following aspiration. Unchanged large air filled structure is noted at the right lung base (arrows).

An additional small pneumothorax was also noted. No abnormal systemic vessels were demonstrated associated with the right basilar anomaly. It was felt that the anomaly likely represented a CPAM and surgical removal was considered. Over the next several days, a follow-up chest $\mathrm{X}$-ray (Figure 4) demonstrated spontaneous resolution of the small residual pneumothorax and the prior visualized right basilar CPAM. Surgical intervention was cancelled.

\section{Discussion}

The renaming of congenital cystic adenomatoid malformation to congenital pulmonary airway malformation has

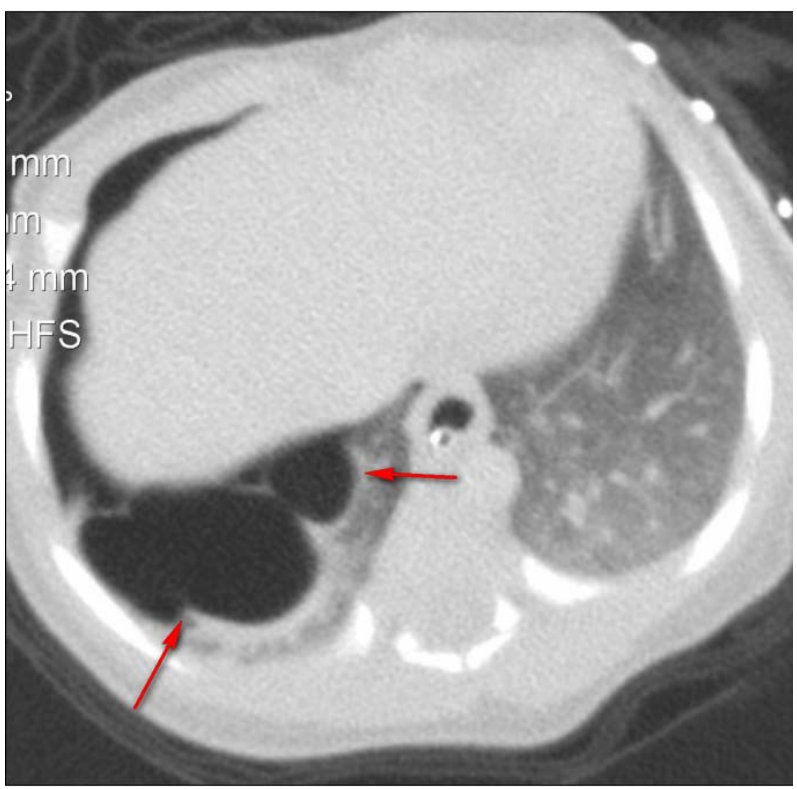

Figure 3. Axial Computed Tomographic image of the chest demonstrate a multilocular air containing structure in the right lower lobe compatible with CРAM (arrows).

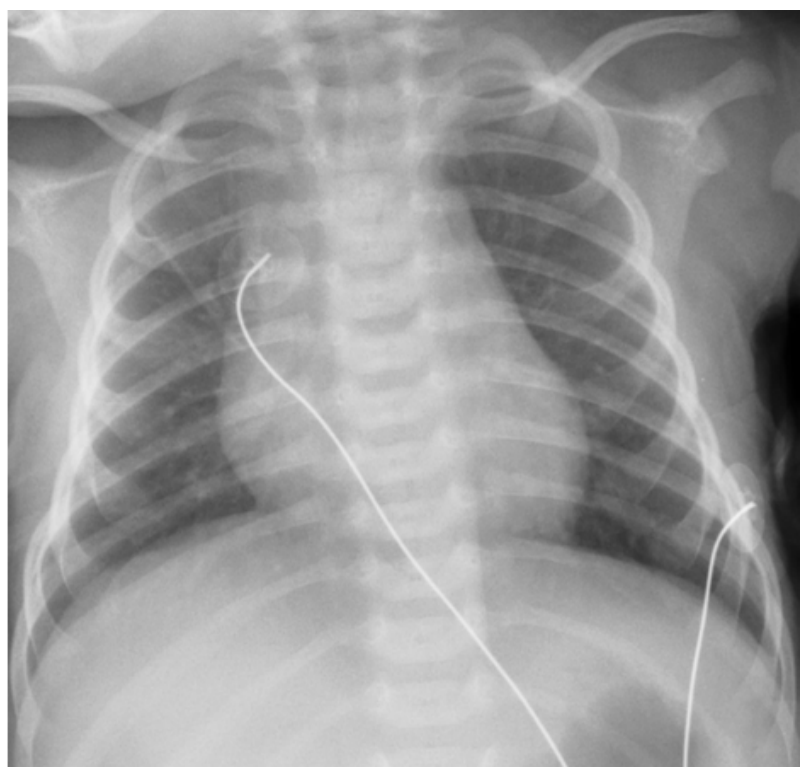

Figure 4. Frontal chest radiograph demonstrates complete resolution of the previously noted right lower lobe CPAM.

occurred within the last ten years. Stocker [4] suggested the term CPAM based on a long experience with the histopathological features of these lesions. Some of the lesions are not "cystic" and others are not adenomatoid. He suggested a reclassification of these lesions based on the anatomic and microscopic features of the pulmonary airways. There are four types of CPAM. Type 0 is a lesion characterized by its involvement with all lobes of the lung, is of tracheal/bronchial origin and represents acinar dysgenesis or dysplasia. Type 0 is incompatible 
with life.

Types 1, 2 and 3 correspond to the original classification of these types, and are of bronchial/bronchiolar, bronchiolar, and bronchiolar/alveolar duct origin, respectively. Type 4 refers to a lesion of distal acinar origin and has a similar radiographic appearance as the original type 1 lesion, except the type 4 lesion appears at the periphery of the lung and has thin walled cysts. Spontaneous tension PTX appears to be a unique feature of the type 4 lesion. There is a controversial association among type 4 CPAMs with pleuralpulmonary blastoma and rhabdomyosarcoma.

We postulate that the CPAM demonstrated in this case report most accurately matches a type 4 lesion, based on its imaging appearance and the clinical association with tension pneumothorax.

Spontaneous PTX in newborn infants has been reported in less than $1 \%$ of all live births [5]. The association of spontaneous PTX in the newborn and congenital major urinary tract anomalies is controversial [5]. Spontaneous PTX resulting from CPAM has been previously reported in a small number of cases [2]. The exact cause of the pneumothorax is not clear but may be the result of "cyst" rupture into the pleural space. As mentioned above, spontaneous PTX is a unique feature of the type 4 lesion, which is usually peripherally based.

The recommended treatment of symptomatic CPAM is surgical resection. However, there is controversy regarding the management of asymptomatic CPAM. Sauvat [6] claimed that surgery may be indicated in asymptomatic CPAM because it will prevent late complications, including pulmonary infection and development of bronchoalveolar carcinoma and rhabdomyosarcoma. However, the risks of surgery and anesthesia in infants and young children must also be considered.

Post-natal spontaneous resolution of CPAM has rarely been reported $[3,6]$. In some cases, the lesion appeared to shrink over time, while in others, the lesion spontaneously resolved. The cause of resolution of CPAM is not clear. The case presented here adds to this experience.

Although we do not have pathological proof of the lesion reported here, we believe that it most likely represent a type 4 CPAM as discussed above. It is unlikely that the lesion represented a loculated pneumothorax, as it appeared at birth, before any intervention had taken place.

Differential diagnosis included congenital pneumatocele, bronchogenic cyst or possibly a pleuralpulmonary blastoma. Pneumatoceles are most frequently of infectious etiology, which was not present in this case. Bronchogenic cysts and pleuralparenchymal blastoma usually do not resolve spontaneously.

In summary, our case report documents a patient who had a spontaneous tension PTX associated with an underlying CPAM, with spontaneous resolution of the CPAM, obviating the need for surgical intervention. It can be added to the literature in which a small number of cases have also been shown to resolve spontaneously in the post-natal period. Therefore, it may be prudent to continue to observe asymptomatic patients with these lesions, as they may resolve post-natally.

\section{REFERENCES}

[1] R. M. Kravitz, "Congenital Malformations of the Lung," Pediatric Clinics of North America, Vol. 41, No. 3, 1994, pp. 453-472.

[2] S. Gardikis, V. Diditis, A. Polychronidis, D. Mikroulis, et al., "Spontaneous Pneumothorax Resulting from Congenital Cystic Adenomatoid Malformation in a Pre-Term Infant: Case Report and Literature Review,” European Journal of Pediatric Surgery, Vol. 12, No. 3, 2002, pp. 195-198. doi:10.1055/s-2002-32733

[3] S. A. Butterworth and G. K. Blair, "Postnatal Spontaneous Resolution of Congenital Cystic Adenomatoid Malformations," Journal of Pediatric Surgery, Vol. 40, No. 5, 2005, pp. 832-834. doi:10.1016/j.jpedsurg.2005.01.051

[4] J. T. Stocker, "Congenital Pulmonary Airway Malformation: A New Name and an Expanded Classification of Congenital Cystic Adenomatoid Malformations of the Lung,” Histopathology, Vol. 41, Suppl. 2, 2002, pp. 424458.

[5] K. Tawill, F. Abu-Ekteish, O. Tamimi, M. Hathal, et al., "Symptomatic Spontaneous Pneumothorax in Term Newborn Infants,” Pediatric Pulmonology, Vol. 37, No. 5, 2004, pp. 443-446. doi:10.1002/ppul.10447

[6] F. Sauvat, J. Michel, A. Benachi, S. Emond and Y. Revillon, "Management of Asymptomatic Neonatal Cystic Adenomatoid Malformations," Journal of Pediatric Surgery, Vol. 38, No. 4, 2003, pp. 548-552. doi:10.1053/jpsu.2003.50119 